\title{
Testosterone Therapy and Cardiovascular Risk
}

\author{
James P. Walsh, M.D., Ph.D. ${ }^{\ddagger *}$ and Anne C. Kitchens, M.D. ${ }^{\ddagger}$
}

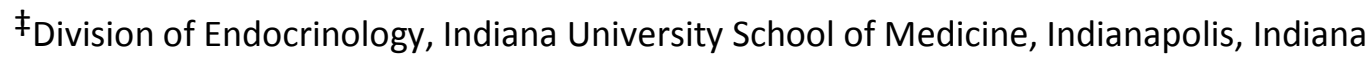

${ }^{\dagger}$ Endocrinology Section, Roudebush Veterans Affairs Medical Center, Indianapolis, Indiana

*Corresponding Author: łEndocrinology Section, Roudebush VAMC, 1481 West Tenth Street, Indianapolis, IN 46202. Tel: 317-988-3073, Fax: 317-988-2641, email: jpwalsh@iu.edu.

The authors have no conflicts of interest to disclose.

This is the author's manuscript of the article published in final edited form as: Walsh, J. P., \& Kitchens, A. C. (2015). Testosterone therapy and cardiovascular risk. Trends in cardiovascular medicine, 25(3), 250-257. http://dx.doi.org/10.1016/j.tcm.2014.10.014 


\begin{abstract}
Endogenous testosterone levels are inversely associated with cardiovascular risk in older men and men with cardiovascular disease. Current data on cardiovascular outcomes of testosterone therapy include only observational studies and adverse event monitoring in short term trials that were not designed to measure cardiovascular outcomes. These studies have yielded conflicting results, and some have raised concerns that testosterone therapy may increase cardiovascular risk. A well designed, adequately powered, prospective trial will ultimately be required to clarify whether testosterone therapy impacts cardiovascular outcomes. This review describes the findings and limitations of recent studies of cardiovascular risk in older men on testosterone therapy and discusses some of the mechanisms through which testosterone may modify cardiovascular risk
\end{abstract}

Key Words: Testosterone; Cardiovascular Disease; Vascular Risk; Thrombosis; Aging. 


\section{Introduction}

Serum testosterone declines gradually as men age. About $20 \%$ of healthy men over age 60 have a total testosterone below the normal range determined in healthy young men (1). The high prevalence of lower testosterone in older men and aggressive marketing of new formulations for nonspecific symptoms have resulted in a marked increase in testosterone prescribing over the last decade (2-4). In addition, men receiving testosterone are often not being diagnosed or monitored in accordance with current guidelines (4). Testosterone replacement in younger men with diseases of the pituitary-gonadal axis is clearly beneficial, but there is little data on the risks or benefits of testosterone in men with agerelated declines in testosterone. Controversy over the risks of testosterone is thus focused on older men without demonstrable pituitary-gonadal disease.

It has been suggested that testosterone may be associated with cardiovascular (CV) risks based on gender differences in atherosclerotic disease and observations of CV events in anabolic steroid abusers (5). More recent studies demonstrating that low testosterone in men is inversely associated with CV risk have led to suggestions that testosterone replacement may be beneficial (6). Safety concerns about testosterone came to the forefront after the recent publication of two observational studies suggesting associations of testosterone therapy with adverse CV outcomes $(7,8)$. These studies were widely covered by the media, and cardiologists are likely to see patients with questions about testosterone and $\mathrm{CV}$ risk. This article reviews testosterone's $\mathrm{CV}$ effects in adult men, focusing on recent clinical studies. While there are concerns that testosterone therapy may increase CV events in older men, limitations of existing data leave significant uncertainty. A well designed, adequately powered, prospective trial of testosterone therapy focused on CV outcomes will be required to address these questions.

\section{Physiology of testosterone actions on the vasculature}


The gonadal axis in adult men is regulated by gonadotropin-releasing hormone (GnRH) from the hypothalamus (Figure 1). GnRH stimulates secretion of luteinizing hormone (LH) and follicle stimulating hormone (FSH) from the anterior pituitary (9). Luteinizing hormone acts on Leydig cells in the testis to stimulate testosterone synthesis, while FSH acts on Sertoli cells in the seminiferous tubules to stimulate spermatogenesis (9). Testosterone binds to cytosolic androgen receptors found in most tissues (10). The testosterone-androgen receptor complex migrates to the nucleus and where it stimulates transcription of numerous genes. There is also evidence for nongenomic testosterone actions through both a membrane-associated form of the classical androgen receptor and a distinct receptor with pharmacologic characteristics of a heterotrimeric $\mathrm{G}$ protein coupled receptor (11).

Testosterone is a prohormone. It is converted to estradiol in many tissues by aromatase, which is expressed in many CV cell types. Circulating estradiol levels in adult men are 10 to $20 \%$ of those in premenopausal women. Testosterone is also converted to dihydrotestosterone by $5 \alpha$-reductase. Circulating dihydrotestosterone levels are lower than those of testosterone, but dihydrotestosterone is a more potent androgen than testosterone and contributes significantly to testosterone's effects. Testosterone, dihydrotestosterone, and estradiol all exert negative feedback at the hypothalamus and pituitary to inhibit LH and FSH release (9).

Most circulating testosterone is bound to sex-hormone binding globulin (SHBG) or albumin (12). Only 1$3 \%$ of total testosterone is not bound to plasma proteins. This unbound, free testosterone is the biologically active hormone fraction. Many clinical conditions alter SHBG concentrations and cause corresponding changes in total testosterone. Obesity, type 2 diabetes, nephrotic syndrome, hypothyroidism, and glucocorticoids decrease SHBG; whereas advanced age, cirrhosis and hepatitis, hyperthyroidism, some anticonvulsants, estrogens, and HIV infection increase SHBG (12). Total testosterone should not be used to diagnose hypogonadism when a condition that alters SHBG is 
present. In this situation, it is important to use free rather than total testosterone. Widely available direct free testosterone assays lack precision (12). A calculated free testosterone is a better marker. Serum testosterone also undergoes diurnal variation driven by nocturnal LH surges from the pituitary. Levels rise overnight and then decline through the day (12). A diagnosis of hypogonadism should be based on testosterone measurements early in the morning when levels are at their peak.

Testosterone and dihydrotestosterone exert numerous CV actions through androgen receptors in endothelium, vascular smooth muscle, and cardiomyocytes (13). Estrogen, which is derived from testosterone, also has direct CV effects. In addition, sex steroids modulate inflammation, coagulation, and other functions that indirectly affect the vasculature $(13,14)$. Rapid CV actions of sex steroids, such as vasodilation, are mediated by nongenomic pathways $(11,15,16)$. Longer term actions occur via testosterone's transcriptional pathway (17). Numerous effects of testosterone on CV physiology have been described; and plausible mechanisms for beneficial or adverse clinical outcomes of testosterone therapy can be proposed $(13,14)$. It is thus difficult to predict effects of testosterone on cardiovascular events based on its physiologic actions. Clinical trials are needed to clarify the CV outcomes of testosterone therapy in older men.

\section{Endogenous Testosterone in Older Men}

In healthy men, total testosterone declines about $10 \%$ per decade after age $40-50$ (18). Low testosterone is also associated with many chronic conditions. Obesity is strongly associated with low testosterone, and levels recover with weight loss (19). Other conditions associated with low testosterone include sleep apnea, type 2 diabetes, hypertension, hyperlipidemia, C-reactive protein and other inflammatory markers, chronic kidney disease, chronic obstructive pulmonary disease, HIV/AIDS, and chronic opioid use (20). Levels of LH and FSH do not increase in response to the declining testosterone and generally remain in the normal range. Many, but not all, studies have found low 
testosterone to be associated with CV disease. A recent meta-analysis of prospective observational trials found inverse associations of testosterone with all-cause and CV mortality (21). Inverse associations with incident CV events have also been noted, but only in studies that included men over 70 years of age (6). The relative risk for an increase of one standard deviation of testosterone was 0.84 (95\% $\mathrm{Cl} 0.76$ to 0.92 ). Another meta-analysis confirmed the association of low testosterone with mortality, but failed to demonstrate a significant association with incident CV disease (22). The point estimate suggested increased CV disease incidence with lower testosterone, but the $95 \% \mathrm{Cl}$ included no effect. In men with chronic heart failure, testosterone is inversely associated with New York Heart Association class, and lower testosterone predicts reduced 3-year survival (23).

Whether decreased testosterone is a cause of adverse clinical outcomes in patients with CV disease and other chronic conditions is unknown. Younger men with low testosterone due to diseases of the pituitary-gonadal axis complain of sexual symptoms, fatigue, mood changes, decreased muscle mass, and increased fat mass (12). These symptoms are common in men with chronic systemic conditions even when testosterone is normal. Conversely, older men with low testosterone are often asymptomatic (24). Testosterone levels in older men thus correlate poorly with symptoms. There is concern that low testosterone in men treated with androgen deprivation for prostate cancer may contribute to an increase in CV events and mortality (25). However, a recent meta-analysis of eight prospective, randomized trials of androgen deprivation for nonmetastatic prostate cancer that reported CV events by study arm did not find evidence for an increase in CV risk (26). Admittedly, findings in men with castrate testosterone levels due to androgen deprivation may not be generalizable to older men with modest reductions in testosterone. Nevertheless, this result does not support a causal relationship between low testosterone and CV events. Overall, the diversity of chronic conditions associated with low testosterone and lack of correlation of testosterone levels with symptoms suggest that low 
testosterone in older men may be more a marker of poor health than a cause of symptoms and clinical outcomes.

\section{Testosterone replacement and cardiovascular events}

It is possible that low endogenous testosterone contributes to CV events and mortality, and that testosterone replacement is beneficial. Alternatively, declines in testosterone with aging and chronic disease could be an adaptive response and testosterone replacement may be harmful. Testosterone replacement in men with symptomatic hypogonadism due to diseases of the pituitary-gonadal axis is not controversial. But it is not clear that these benefits translate to men with functional declines in testosterone related to chronic diseases or aging. The only data available are retrospective studies and adverse event reporting from short term testosterone trials that were not designed to measure CV outcomes. Major recent studies of testosterone therapy and CV events or mortality are summarized in Table 1. As described below, these studies have yielded conflicting results.

Two retrospective studies have found testosterone replacement in men with low endogenous testosterone to be associated with improved survival. Shores et al. examined mortality in 1,031 U.S. veterans with total testosterone less than $8.7 \mathrm{nmol} / \mathrm{L}(250 \mathrm{ng} / \mathrm{dL}), 398$ of whom were initiated on testosterone therapy in the course of routine care (27). The average follow up duration was 40.5 months. The median time from testosterone measurement to initiation of testosterone treatment was 3.3 months and the mean duration of treatment was 20.2 months. Once treatment was initiated, subjects were classified as treated for the duration of follow up. The baseline prevalence of coronary disease was $21.9 \%$. Overall mortality in testosterone treated men was $10.3 \%$, versus $20.7 \%$ in untreated men. In a Cox regression model with testosterone as a time varying exposure and adjusted for nine covariates, testosterone replacement was associated with a decreased risk of death, hazard ratio $0.61, p$ $=0.008$. If men who stopped treatment were censored 90 days after their last testosterone refill, the 
hazard ratio increased slightly to 0.65 , but the $p$-value was no longer significant. No information was provided on causes of death. In the other study, Muraleedharan et al. retrospectively reviewed 581 men with type 2 diabetes who had testosterone measured (28). Subjects were followed for a mean of 6 years. Of the $581 \mathrm{men}, 238 \mathrm{had}$ a testosterone level less than $10.4 \mathrm{nmol} / \mathrm{L}$ ( $300 \mathrm{ng} / \mathrm{dL}$ ), and 64 received testosterone therapy for an average of 41.6 months. In a Cox regression model, the hazard ratio for mortality in the treated group versus the group with untreated low testosterone was $0.43, p=0.004$. The survival curve for treated patients was similar to that of patients with normal testosterone. Causes of death by treatment group were not reported.

Two other retrospective studies found testosterone therapy to be associated with increased CV risk. Vigen et al. examined a composite outcome of mortality, myocardial infarction, and stroke in male U.S. veterans who had coronary angiography and a total testosterone less than $10.4 \mathrm{nmol} / \mathrm{L}(300 \mathrm{ng} / \mathrm{dL})$ (7). Of the 8,709 veterans in the cohort, 1,223 were initiated on testosterone therapy a median of 531 days after coronary angiography. Average follow up was 840 days. Once initiated on testosterone, patients were assumed to continue on therapy. However, $17.6 \%$ of these men filled only one prescription and the average duration of therapy for men who filled multiple prescriptions was 376 days. There were 123 events in the testosterone group (10.1\%) versus 1,587 events in the untreated men (21.2\%). The prevalence of coronary disease was $87.4 \%$. Untreated men were older and had higher rates of numerous comorbidities including coronary disease. In a Cox regression model with testosterone as a time-varying exposure and weighted for over 50 other covariates, the hazard ratio for an event on testosterone was $1.29, p=0.02$. This result was not modified by the presence of coronary disease. However, the number of subjects without coronary disease was small. In the other study, Finkle et al. examined the risk of nonfatal myocardial infarction (MI) in 55,593 men in a large insurance claims database who filled a first testosterone prescription between 2006 and 2010 (8). The event rate in the 90 days following testosterone initiation was compared to the rate in the previous year. The post/pre- 
prescription ratio of acute $\mathrm{MI}$ incidence was 1.36 (95\% $\mathrm{Cl} 1.03-1.81)$. Excess post-prescription $\mathrm{MI}$ risk was only seen in men over 65 or with preexisting heart disease. An identical analysis was performed on 167,279 men in the same database who filled a first prescription for a type 5 phosphodiesterase inhibitor, a drug class with well-established CV safety. After odds-of-treatment weighting to adjust the distribution of covariates to that in the testosterone cohort, the post/pre-prescription acute MI incidence ratio was 1.08 (95\% $\mathrm{Cl} 0.93-1.24)$. There was no evidence of increased MI risk with phosphodiesterase inhibitors in patients over 65 or with preexisting heart disease.

While each of these studies adjusted for likely confounders, residual confounding is an inherent limitation of retrospective studies. Providers may elect not to offer testosterone to more severely ill patients, and such patients may be less inclined to request testosterone replacement. Another potential bias is the common practice of prescribing testosterone for erectile dysfunction, a known predictor of CV disease. In the Shores, Muraleedharan, and Vigen studies, the average duration of testosterone treatment was substantially less than the follow up period, implying a high rate of treatment discontinuation. Initial indications for treatment and reasons for discontinuation were not available. Only total testosterone levels were reported, presumably because more biologically relevant free testosterones were not available. Testosterone levels were also not consistently measured in the morning. Use of total testosterone and inclusion of testosterone levels measured later in the day likely resulted in inclusion of men with normal free testosterone. The Finkle study attempted to address some of these issues by comparing cardiovascular events over the year prior to testosterone therapy to the 90 day period after therapy was initiated, effectively using each subject as his own control. However, the lack of baseline information on the patients, including testosterone levels and other indications for therapy remains a significant limitation. 
The only prospective data on CV risks of testosterone comes from adverse event monitoring in randomized trials. With the exception of polycythemia, discussed below, most trials have not noted significant differences in CV events between treatment arms. This changed in 2010 when the Testosterone in Older Men with Mobility Limitations (TOM) trial was terminated early due to excess CV events in the testosterone group (29). The trial had enrolled 209 men at the time it was halted. The TOM trial was following measures of physical performance in men over 65 with limited mobility and total testosterone between 3.5 and $12.1 \mathrm{nmol} / \mathrm{L}$ (100 to $350 \mathrm{ng} / \mathrm{dL}$ ) or free testosterone less than 173 $\mathrm{pmol} / \mathrm{L}(50 \mathrm{pg} / \mathrm{mL})$. Cardiovascular events occurred in 23 men in the testosterone group and 5 in the placebo group. The odds ratio for CV events was 5.4 (95\% Cl 2.0-14.9) and changed only slightly after adjustment for several risk factors. The risk of an event was constant over the 24 week intervention period. In a subsequent analysis, the increase in free testosterone on therapy was significantly associated with the risk of an event (30). Participants in the TOM trial were older and had a higher prevalence of chronic conditions than most other trials of testosterone therapy. The testosterone doses used were often higher than are typically used for replacement therapy. Events observed included serious events such as myocardial infarction, stroke, congestive heart failure, and atrial fibrillation. However they also included less severe events such as peripheral edema, premature ventricular contractions, elevated blood pressure, and others. Testosterone would need to act through multiple mechanisms to cause such diverse outcomes. However, existence of multiple mechanisms is not implausible given the pleiotropic CV actions of testosterone discussed above. Subjects in the treatment arm were also referred for evaluation of some noncardiovascular conditions more frequently than controls. This raises the possibility of an ascertainment bias, as some CV events may have been discovered incidentally during evaluation of another complaint.

A 2013 meta-analysis by Xu et al. of 27 testosterone trials that reported CV events by study arm found an odds ratio for events on testosterone therapy of 1.54 (95\% $\mathrm{Cl} 1.09$ to 2.18$)$ (31). Cardiovascular risk 
on therapy did not depend on baseline testosterone, but did vary with the source of funding. In 13 industry-funded studies, testosterone had no effect. In the 14 studies not funded by industry, the odds ratio for an event on therapy was 2.06 (95\% Cl 1.34 to 3.17). Men in the industry-sponsored trials were younger and had a lower overall event rate, so this difference may reflect lower baseline risk. One limitation of this meta-analysis is variability among the trials in severity of adverse events reported. However a similar odds ratio, 1.61 (95\% $\mathrm{Cl} 1.01$ to 2.56$)$, was seen when the analysis was limited to serious events. The point estimate of the odds ratio for CV death in the testosterone arms was similar at 1.42, but the $95 \% \mathrm{Cl}$ included no effect. A 2014 meta-analysis by Corona et al. identified 75 randomized trials of testosterone therapy that reported CV events by study arm and examined a composite outcome of CV death, acute myocardial infarction, stroke, acute coronary syndromes, and heart failure (32). However, this total included many zero-event trials that were excluded from the main analysis, which was performed on 26 trials. The odds ratio for an event on testosterone was 1.01 ( $95 \% \mathrm{Cl} 0.57$ to 1.77 ). The Corona study included 5 trials that were excluded by Xu et al., while the Xu study included 6 trials that were excluded by Corona et al. Limitations of both of these meta-analyses include the fact that the trials were not stratified by CV risk and did not measure CV events as prespecified outcomes. Many of the trials were limited to subjects with specific conditions such as rheumatoid arthritis, HIV/AIDS, cirrhosis, heart failure, COPD, and malnutrition, which may limit the generalizability of the results. It is also important to note that all of the included studies were for short durations, 6 weeks to 36 months. There is no good clinical trial data on long term risks of testosterone therapy.

The mechanisms through which testosterone may modify CV risk are poorly understood. Physiologic testosterone replacement in men with modestly reduced testosterone slightly decreases HDL and has little effect on other lipid fractions $(33,34)$. There is no effect on systolic or diastolic blood pressure (34). Testosterone trials in men with type 2 diabetes or metabolic syndrome have consistently demonstrated favorable changes in adiposity and insulin resistance, and some have found improvements hemoglobin 
A1c, fasting glucose, and other markers of the metabolic syndrome $(19,35)$. These observations have led to proposals that testosterone-induced improvements in metabolic risk might contribute to reductions in cardiovascular disease and other diabetic complications. However no prospective trial to test this hypothesis has been conducted. There is evidence that testosterone therapy improves exercise capacity in men with congestive heart failure (36). A recent meta-analysis of four randomized trials of testosterone therapy in heart failure enrolling a total of 198 subjects found that testosterone treatment improved exercise capacity in the 6-minute walk test by 54 meters $(95 \% \mathrm{Cl} 43-65)$ or the incremental shuttle walk test by 47 meters $(95 \% \mathrm{Cl} 13-81)$. Peak oxygen consumption was also significantly increased. Treatment durations ranged from 12 weeks to 12 months. None of the trials showed improvements in left ventricular ejection fraction measured by echocardiography, and the authors suggested that these improvements occurred via peripheral mechanisms. Too few clinical events were observed to draw conclusions about CV safety. There is also evidence that testosterone may improve myocardial ischemia. Two randomized trials and one crossover trial examined the effect of testosterone treatment for 4 to 52 weeks on ST-segment depression during treadmill exercise testing in a total of 69 men (37-39). The three studies observed significantly longer times to $1 \mathrm{~mm}$ ST-segment depression, ranging from 59 to 109 seconds, in testosterone versus placebo-treated men. The effect was greater in subjects with lower baseline testosterone levels. The authors speculated that the results reflect a vasodilatory effect of testosterone on the coronary vasculature based on similar findings after acute intravenous testosterone infusion in other studies. There was only one serious adverse event in the three trials and no conclusions regarding CV safety could be drawn.

A well-described effect of testosterone that may contribute to CV events is erythrocytosis. Testosterone therapy causes a $3.2 \%$ absolute increase in hematocrit, and polycythemia is sometimes seen (34). High hematocrit is associated with increased CV risk in observational studies, and may contribute to events in men using testosterone (40). A recent report suggested an association of venous thrombosis with 
testosterone therapy. Glueck and coworkers identified 42 patients with thrombotic events a median of 4.5 months after initiating testosterone therapy, 39 of whom were shown to have a hereditary thrombophilia (41). The authors speculated that the events were attributable to estradiol formed by aromatization of the exogenous testosterone. Estrogen therapy in women with hereditary thrombophilia markedly increases the risk of venous thrombosis (42). However, estrogen levels in these women are higher than those in men using testosterone, and much of the risk with estrogen may be attributable to hepatic first pass effects of oral preparations, an effect which would not occur with testosterone therapy (43). Nevertheless, men may be more sensitive to thrombotic effects of estradiol, and testosterone may have other actions that predispose men to thrombosis. These observations require confirmation in a prospective trial. There is also evidence that myocardial infarction is associated with hereditary thrombophilia, suggesting that if an association of testosterone with venous thrombosis is confirmed, testosterone could increase the risk of arterial thrombosis as well (44).

In spite of their limitations, the recent reports of CV events in older men on testosterone therapy raise significant concern that testosterone may be associated with adverse CV outcomes. After publication of the Vigen and Finkle studies, the Endocrine Society issued a position statement recommending that testosterone only be prescribed in accordance with established guidelines, and only after discussion of possible CV risks with the patient (45). The U.S. Food and Drug Administration recently required that all testosterone products include a general warning about venous thrombosis (46). Until better data on the long term safety are available, a conservative approach to testosterone replacement in men with age and comorbidity-related declines in testosterone is warranted.

\section{What a cardiologist needs to know}

With many older men now using testosterone and extensive media coverage of potential CV risks, cardiologists will see patients with questions about the safety of testosterone (47). Guidelines for 
testosterone therapy have been developed by several societies $(12,48)$. A full discussion of the considerations for initiation and monitoring of testosterone replacement is beyond the scope of this article. There is considerable overlap of testosterone levels in hypogonadal men with those in asymptomatic, healthy men. As a result, there is no consensus threshold for diagnosis of hypogonadism based on a laboratory value, and all guidelines emphasize the importance of interpreting a testosterone level in the context of the patient's symptoms. Due to intraindividual fluctuations, low testosterone levels should also be confirmed with a repeat morning measurement. One guideline suggests that levels between 8 and $12 \mathrm{nmol} / \mathrm{L}(231-346 \mathrm{ng} / \mathrm{dL})$ are borderline and require further investigation (48). The Endocrine Society guideline simply notes that the lower limit of normal in young healthy men is usually 9.7 to $10.4 \mathrm{nmol} / \mathrm{L}(280-300 \mathrm{ng} / \mathrm{dL})(12)$. There is significant variability among assays and it is recommended that practitioners consider the lower end of the normal range established by the laboratory they are using. Given the high prevalence of conditions that alter SHBG in men with CV disease, it is our practice to base treatment decisions on calculated free testosterone rather than total testosterone. The testosterone level should be monitored and the dose titrated to keep testosterone at or below the middle of the normal range for younger men. Testosterone should be discontinued if there is no improvement in the symptoms being attributed to hypogonadism after a 4 to 6 month trial. Until future studies clarify the CV risks of testosterone, it is also recommended that providers discuss possible vascular risks with patients considering testosterone replacement. Specific contraindications to testosterone therapy include polycythemia, untreated obstructive sleep apnea, and poorly controlled heart failure, as testosterone may worsen these conditions (12). Prostate cancer, unevaluated prostate nodules or prostate specific antigen elevations, severe lower urinary tract symptoms, and breast cancer are also contraindications. Hematocrit, digital rectal exam, and prostate specific antigen should be monitored in men using testosterone. Until better data on long term safety is available, cautious use of testosterone is recommended for older men and men with cardiovascular disease. 


\section{Summary and future directions}

Millions of older men are now using testosterone for age related declines in serum testosterone and nonspecific symptoms. The data on cardiovascular risks of testosterone in this population have significant limitations. Observational studies have produced conflicting results and no prospective trial of testosterone therapy has examined CV events as a prespecified outcome. There is no data on long term risks. A few ongoing trials are examining the effect of testosterone replacement on markers of atherosclerosis progression (NCT00799617, NCT00287586, NCT00467987) or heart failure (NCT01813201, NCT01852994), but none of these studies are powered to assess differences in CV events. In the absence of more definitive data, performing structured, consistent analyses of CV events in testosterone trials may facilitate firmer conclusions in a future meta-analysis (49). Future trials should also include more biologically relevant free testosterone levels, not just total testosterone, and should always measure endogenous testosterone in the morning. For patients with hypogonadism from diseases of the pituitary gonadal axis, testosterone cures their symptoms and improves quality of life. For men with age or chronic disease-related declines of testosterone, judicious use of testosterone therapy is preferred. Given the conflicting findings of existing reports, additional retrospective studies are unlikely to resolve the controversy over testosterone therapy in older men. A well designed, adequately powered, prospective trial of sufficient duration will ultimately be needed to determine if testosterone modifies cardiovascular risk. 


\section{References}

1. Harman SM, Metter EJ, Tobin JD, Pearson J, Blackman MR. Longitudinal effects of aging on serum total and free testosterone levels in healthy men. J Clin Endocrinol Metab 2001;86:72431.

2. Braun SA. Promoting "low-T", a medical writer's perspective. JAMA Int Med 2013;173:1458-60.

3. Baillargeon J, Urban RJ, Otenbacher KJ, Pierson KS, Goodwin JS. Trends in androgen prescribing in the United States, 2001 to 2011. JAMA Int Med 2013;173:1465-6.

4. Layton JB, Li D, Meier CR, Sharpless JL, Sturmer T, Jick SS, et al. Testosterone lab testing and initiation in the United Kingdom and the United States, 2000 to 2011. J Clin Endocrinol Metab 2014;99:835-42.

5. Pope HG, Wood RI, Rogol, A, Nyberg F, Bowers L, Bhasin S. Adverse health consequences of performance enhancing drugs: An Endocrine Society scientific statement. Endocr Rev 2014;35:341-75.

6. Ruige JB, Mahmoud AM, De Bacquer D, Kaufman JM. Endogenous testosterone and cardiovascular disease in healthy men: a meta-analysis. Heart 2011;97:870-75.

7. Vigen R, O'Donnell Cl, Baron AE, Grunwald GK, Maddox TM, Bradley SM, et al. Association of testosterone therapy with mortality, myocardial infarction, and stroke in men with low testosterone levels. JAMA 2013;310: 1829-36.

8. Finkle WD, Greenland S, Ridgeway GK, Adams JL, Frasco MA, Cook MB, Fraumeni JF, Hoover RN. Increased risk of non-fatal myocardial infarction following testosterone therapy prescription in men, PLoS One 2014;9:e85805.

9. Reyes-Fuentes A, Veldhuis JD. Neuroendocrine physiology of the normal male gonadal axis. Endocrinol Metab Clin North Am 1993;22:93-124.

10. Mooradian AD, Morley JE, Korenman SG. Biological actions of androgens. Endocr Rev 1987;8:128.

11. Rahman F, Christian HC. Non-classical actions of testosterone: an update. Trends Endocrinol Metab 2007;18:371-8.

12. Bhasin S, Cunningham GR, Hayes FJ, Matsumoto AM, Snyder PJ, Swerdloff RS, et al. Testosterone therapy in adult men with androgen deficiency syndromes: an Endocrine Society clinical practice guideline. J Clin Endocrinol Metab 2010;95:2536-59.

13. Ruige JB, Ouwens M, Kaufman JM. Beneficial and adverse effects of testosterone on the cardiovascular system in men. J Clin Endocrinol Metab 2013;98:4300-10.

14. Herring MJ, Oskui PM, Hale SL, Kloner RA. Testosterone and the cardiovascular system: a comprehensive review of the basic science literature. J Am Heart Assoc 2013;2:e000271.

15. Chambliss KL, Shaul PW. Estrogen modulation of endothelial nitric oxide synthase. Endocr Rev 2002;23:665-86.

16. Deenadayalu V, Puttabyatappa Y, Liu AT, Stallone JN, White RE. Testosterone-induced relaxation of coronary arteries: activation of BKCa channels via the cGMP-dependent protein kinase. Am J Physiol Heart Circ Physiol 2012;302:H115-23.

17. Eyster, KM, Mark CJ, Gayle R, Martin DS. The effects of estrogen and testosterone on gene expression in rat mesenteric arteries. Vascul Pharmacol 2007;47:238-47.

18. Travison TG, Araujo AB, Kupelian V, O'Donnell AB, McKinlay JB. The relative contributions of aging, health, and lifestyle factors to serum testosterone decline in men. J Clin Endocrinol Metab. 2007;92:549-55.

19. Grossman M. Low testosterone in men with type 2 diabetes: significance and treatment. J Clin Endocrinol Metab 2011;96:2341-53. 
20. Kalyani RR, Gavini S, Dobs AS, Male hypogonadism in chronic disease. Endocrinol Metab Clin N Am 2007;36:333-48.

21. Araujo AB, Dixon JM, Suarez EA, Murad MH, Guey LT, Wittert GA. Endogenous testosterone and mortality in men: a systematic review and meta-analysis. J Clin Endocrinol Metab 2011;96:300719.

22. Corona G, Rastrelli G, Monami M, Guay A, Buvat J, Sforza A, et al. Hypogonadism as a risk factor for cardiovascular mortality in men: a meta-analytic study. Eur J Endocrinol 2011;165:687-701.

23. Jankowska EA, Biel B, Majda J, Szklarska A, Lopuszanska M, Medras M, Anker SD, Banasiak W, Poole-Wilson PA, Ponikowski P. Anabolic deficiency in men with chronic heart failure: prevalence and detrimental impact on survival. Circulation 2006;114:1829-37.

24. Basaria, S. Testosterone therapy in older men with late-onset hypogonadism: a counterrationale. Endocr Pract 2013;19:853-63.

25. Levine GN, D'Amico AV, Berger P, Clark PE, Eckel RH, Keating NL, et al. Androgen-deprivation therapy in prostate cancer and cardiovascular risk: a science advisory from the American Heart Association, American Cancer Society, and American Urological Association: endorsed by the American Society for Radiation Oncology. Circulation 2010;121:833-40.

26. Nguyen PL, Je Y, Schutz FAB, Hoffman KE, Hu JC, Parekh A, et al. Association of androgen deprivation therapy with cardiovascular death in patients with prostate cancer. JAMA 2011;306:2359-66.

27. Shores MM, Smith NL, Forsberg CW, Anawalt BD, Matsumoto AM. Testosterone treatment and mortality in men with low testosterone levels. J Clin Endocrinol Metab 2012;97:2050-8.

28. Muraleedharan V, Marsh H, Kapoor D, Channer KS, Jones TH. Testosterone deficiency is associated with increased risk of mortality and testosterone replacement improves survival in men with type 2 diabetes. Eur J Endocrinol 2013;169:725-33.

29. Basaria S, Coviello AD, Travison TG, Storer TW, Farwell WR, Jette AM, et al. Adverse events associated with testosterone administration. N Engl J Med 2010;363:109-22.

30. Basaria S, Davda MN , Travison TG, Ulloor J, Singh R, Bhasin S. Risk Factors Associated With Cardiovascular Events During Testosterone Administration in Older Men With Mobility Limitation. J Gerontol A Biol Sci Med Sci 2013;68:153-60.

31. Xu L, Freeman G, Cowling BJ, Schooling CM. Testosterone therapy and cardiovascular events among men: a systematic review and meta-analysis of placebo controlled randomized trials. BMC Medicine 2013;11:108.

32. Corona G, Maseroli E, Rastrelli G, Isidori AM, Sforza A, Mannucci E, et al. Cardiovascular risk associated with testosterone-boosting medications: a systematic review and meta-analysis. Expert Opin Drug Saf 2014;13:1327-51.

33. Haddad RM, Kennedy CC, Caples SM, Tracz MJ, Bolona ER, Sideras K, et al. Testosterone and cardiovascular risk in men: a systematic review and meta-analysis of randomized placebocontrolled trials. Mayo Clin Proc 2007;82:29-39.

34. Frenandez-Balsells MM, Murad MH, Lane M, Lampropulos JF, AlbuquerqueF, MullanRJ, et al. Adverse effects of testosterone therapy in adult men: a systematic review and meta-analysis. J Clin Endocrinol Metab 2010;95:2560-75.

35. Jones TH. Effects of testosterone on type 2 diabetes and components of the metabolic syndrome. J Diabetes 2010;2:146-56.

36. Toma M, McAlister FA, Coglianese EE, Vidi V, Vasaiwala S, Bakal JA, et al. Testosterone supplementation in heart failure: a meta-analysis. Circ Heart Fail 2012;5:315. 
37. English KM, Steeds RP, Jones TH, Diver MJ, Channer KS. Low-dose transdermal testosterone therapy improves angina threshold in men with chronic stable angina: a randomized, doubleblind, placebo-controlled study. Circulation 2000;102:1906-11.

38. Malkin CJ, Pugh PJ, Morris PD, Kerry KE, Jones RD, Jones TH, et al. Testosterone replacement in hypogonadal men with angina improves ischaemic threshold and quality of life. Heart 2004;90:871-6.

39. Mathur A, Malkin C, Saeed B, Muthusamy R, Jones TH, and Channer K. Long-term benefits of testosterone replacement therapy on angina threshold and atheroma in men. Eur J Endocrinol 2009;161:443-9.

40. Brown DW, Giles WH, Croft JB. Hematocrit and the risk of coronary heart disease mortality. Am Heart J 2001;142:657-63.

41. Glueck CJ, Wang P. Testosterone therapy, thrombosis, thrombophilia, cardiovascular events. Metabolism 2014;63:989-94.

42. Wu O, Robertson L, Langhorne P, Twaddle S, Lowe GDO, Clark P, et al. Oral contraceptives, hormone replacement therapy, thrombophilias and risk of venous thromboembolism: a systematic review. Thromb Haemost 2005;94:17-25.

43. Olie V, Canonico M, Scarabin PY. Risk of venous thrombosis with oral versus transdermal estrogen therapy among postmenopausal women. Curr Opin Hematol 2010;17:457-63.

44. Mannucci PM, Asselta R, Duga S, Guella I, Spreafico M, Lotta L, et al. The association of factor V Leiden with myocardial infarction is replicated in 1880 patients with premature disease. Thromb Haemost 2010;8:2116-21.

45. Endocrine Society. The risk of cardiovascular events in men receiving testosterone therapy. February 7, 2014. Accessed August 30, 2014. http://www.endocrine.org/advocacy-andoutreach/position-statements/other-society-statements.

46. U. S. Food and Drug Administration. Testosterone products: FDA/CDER statement - risk of venous blood clots. June 20, 2014, Accessed August 30, 2014. http://www.fda.gov/safety/ medwatch/safetyinformation/safetyalertsforhumanmedicalproducts/ucm402054.htm.

47. Von Drehle, D (2014, August 18). Manopause!? Aging, insecurity and the \$2 billion testosterone industry. Time, U.S. Edition 184:36-43.

48. Wang C, Nieschlag E, Swerdloff R, Behre HM, Hellstrom WJ, Gooren L, et al. Investigation, treatment, and monitoring of late-onset hypogonadism in males: ISA, ISSAM, EAU, EAA, and ASA Recommendations. J Androl 2009;30:1-9.

49. Basaria S. Need for standardizing adverse event reporting in testosterone trials. Evid Based Med 2014;19:32-3. 
Table 1

\section{Studies of Cardiovascular Events in Men on Testosterone Therapy}

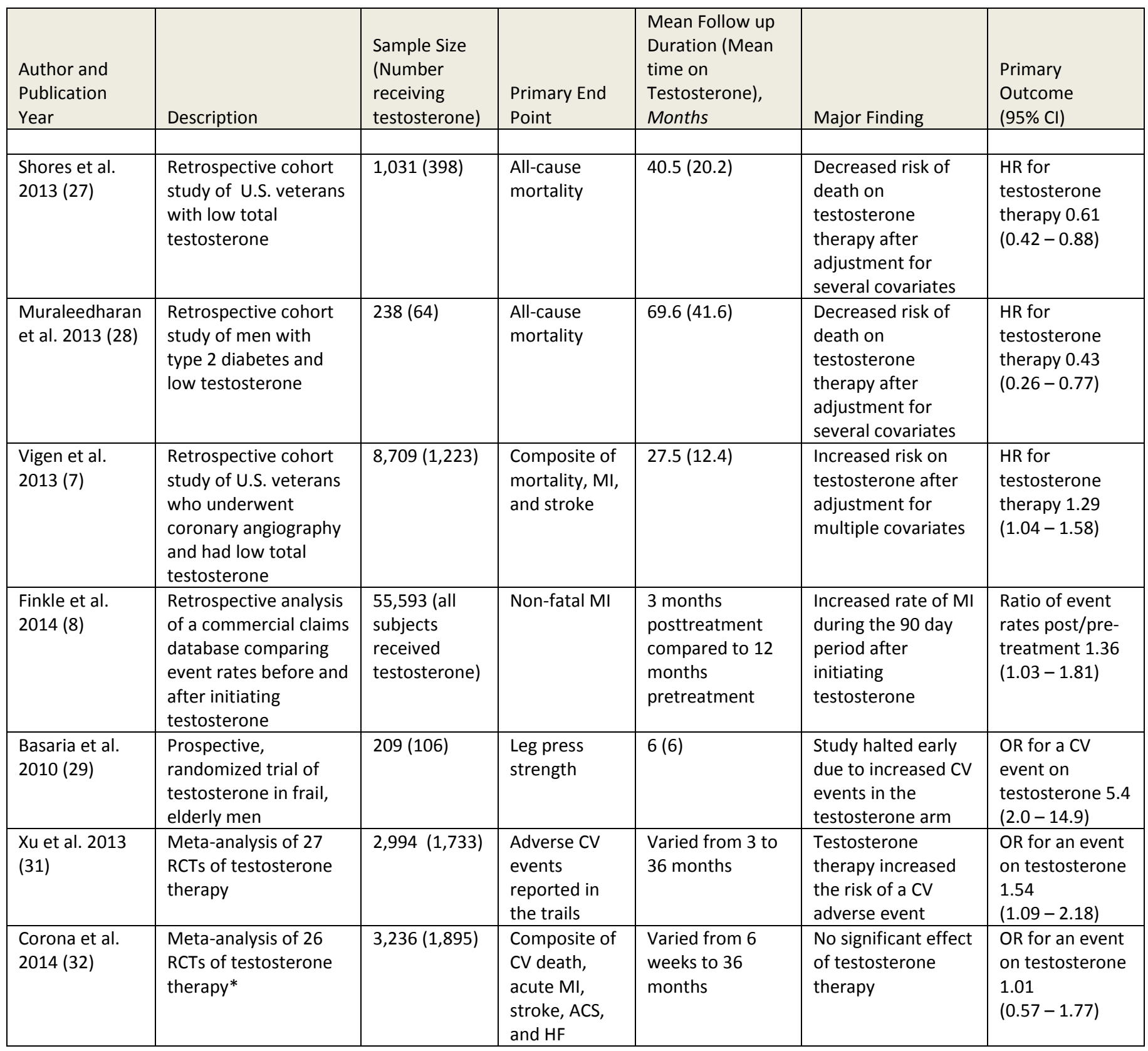

*The meta-analysis by Corona et al. identified 75 trials, but included only 26 in the main analysis. The numbers in the table reflect trials included in the main analysis.

Abbreviations: ACS, acute coronary syndrome; CV, cardiovascular; $\mathrm{HF}$, heart failure; $\mathrm{HR}$, hazard ratio; $\mathrm{OR}$, odds ratio; $\mathrm{MI}$, myocardial infarction; $\mathrm{RCT}$, randomized controlled trial. 


\section{Figure Legends}

Figure 1: Regulation of the pituitary-gonadal axis in adult men. The pituitary is stimulated by gonadotropin releasing hormone $(\mathrm{GnRH})$ from the hypothalamus to secrete leutenizing hormone (LH) and follicle stimulating hormone (FSH). Leutenizing hormone stimulates testosterone synthesis in the Leydig cells of the testis, while FSH stimulates spermatogenesis. Testosterone is converted in peripheral tissues to two other hormones, dihydrotestosterone (DHT) and estradiol. Testosterone, DHT, and estradiol all feed back to the hypothalamus and pituitary to inhibit secretion of GnRH, LH, and FSH. 
Figure 1

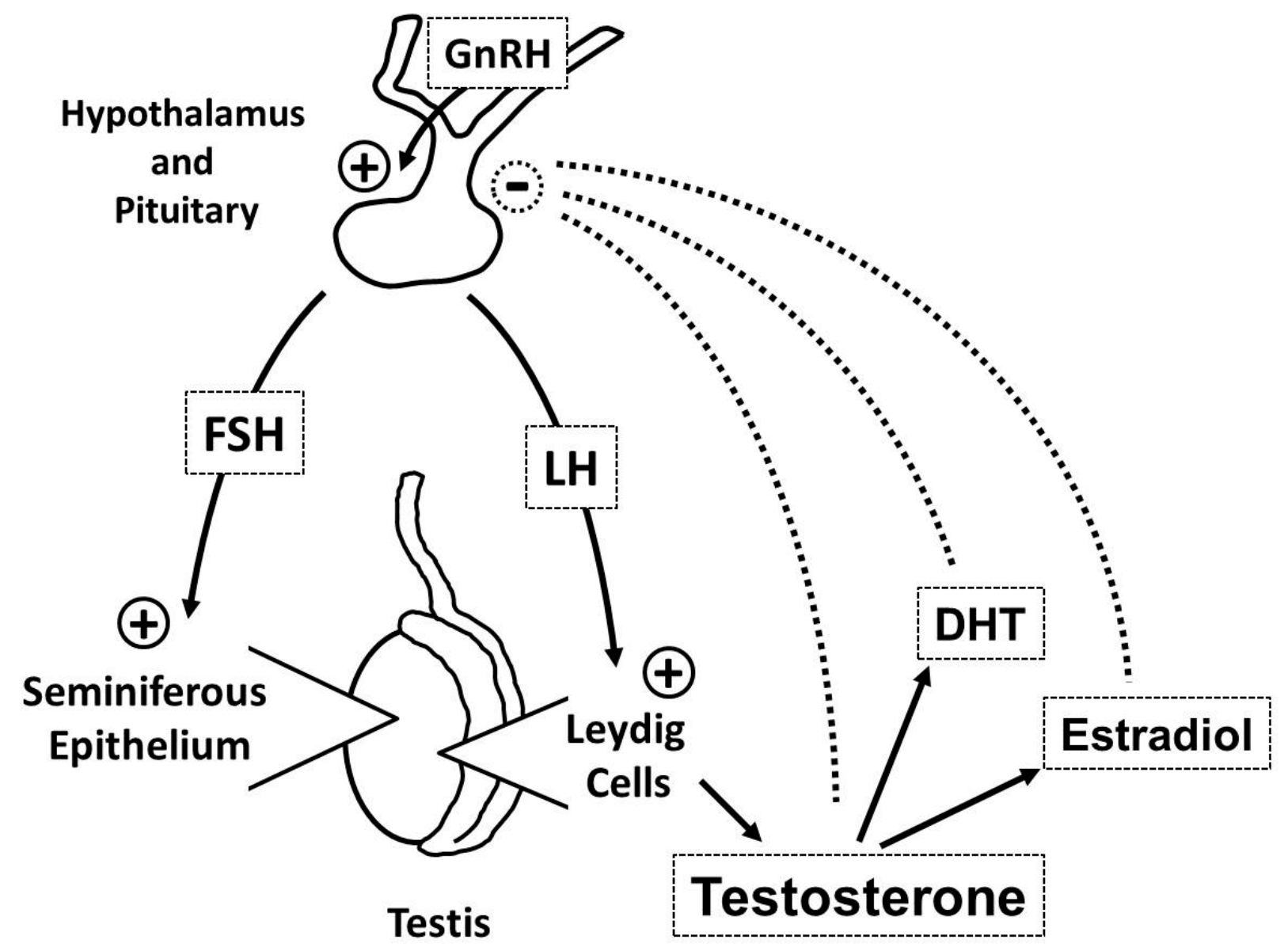

